\title{
The Hemispheric Helicity Rule during the Rise of Cycle 23
}

\author{
S.D. Bao, G.X. Ai, and H.Q. Zhang \\ Beijing Astronomical Observatory/National Astronomical Observatories, \\ Chinese Academy of Sciences, Beijing 100012, China
}

In the last decade observations have revealed that a hemispheric preference of magnetic chirality (handedness) exists throughout the solar atmosphere, such as the net helicity of active regions in the photosphere, filament channels, Sor inverse S-shaped coronal loops and interplanetary magnetic clouds. The explanation of all these patterns and their possible connection to the dynamo are open to question. The aim of this paper is to examine whether the hemispheric sign rule for helicity in active regions does not change with a new solar cycle.

We choose two different current helicity parameters (i.e., $\alpha_{\text {best }}$ and $H_{c}$ ) to compute their signs for 87 active regions during the rising phase of cycle 23 , using the vector magnetograms from the Solar Magnetic Field Telescope of Huairou Solar Observing Station of Beijing Astronomical Observatory. We find that 59\% of the computed active regions in the northern hemisphere have negative $\alpha_{\text {best }}$ and $65 \%$ in the southern hemisphere have positive $\alpha_{\text {best }}$. This result is consistent with that of the cycle 22 . However, the helicity parameter $H_{c}$ shows a weaker opposite hemispheric preference in the current solar cycle.

How to explain the above result? Our views are that: (1) Although such a primary result is interesting, it would be premature to make a serious conclusion based on our data. More samples are required to answer this question. (2) Since Faraday rotation will produce a counterclockwise rotation of the azimuth for a line-of-sight field of positive polarity, and during cycle 23 the polarity of leading sunspots is positive in the northern hemisphere, some active regions with negative current helicity in the northern hemisphere due to the effect of Faraday rotation may reverse their original twist configuration to have positive helicity. For all the active regions in which the original twist is right-hand (positive helicity) in the northern hemisphere, their signs will not be influenced by Faraday rotation. On the other hand, $H_{\mathrm{c}}$ may be more susceptible to Faraday rotation than $\alpha_{\text {best }}$ because it is mainly related to the areas where the line-of-sight field is strong. $H_{\mathrm{c}}$, therefore, has a lower percentage of helicity sign in the northern hemisphere during the cycle 23 than $\alpha_{\text {best }}$. Since the leading sunspots have negative polarity in the northern hemisphere during cycle $22, H_{\mathrm{c}}$ should have a higher percentage in sign than $\alpha_{\text {best }}$. (3) The cause of the hemispheric tendency is still uncertain. Perhaps these two parameters reflect different physical nature of sub-photospheric origin. (4) A single sign of $\alpha_{\text {best }}$ or $H_{\mathrm{c}}$ averaged over a whole active region is not appropriate. It ignores the small-scale patterns of oppositely directed twists which are known to exist inside active regions. More details about this topic have been described by Bao et al. (2000, Astrophysics and Astronomy, in press). 